This item was submitted to Loughborough's Research Repository by the author.

Items in Figshare are protected by copyright, with all rights reserved, unless otherwise indicated.

\title{
Aggregation in colloidal suspensions and its influence on the suspension viscosity
}

PLEASE CITE THE PUBLISHED VERSION

http://dx.doi.org/10.1134/S1061933X10030117

\section{PUBLISHER}

Original Russian Edition Copyright $\odot 2010$ by the Russian Academy of Sciences and the Frumkin Institute of Physical Chemistry and Electrochemistry, Russian Academy of Sciences (@ Pleiades Publishing, Ltd)

\section{VERSION}

AM (Accepted Manuscript)

\section{LICENCE}

CC BY-NC-ND 4.0

\section{REPOSITORY RECORD}

Kovalchuk, Nina, I. Kuchin, Victor Starov, and N. Uriev. 2010. "Aggregation in Colloidal Suspensions and Its Influence on the Suspension Viscosity”. figshare. https://hdl.handle.net/2134/6612. 
This item was submitted to Loughborough's Institutional Repository (https://dspace.lboro.ac.uk/) by the author and is made available under the following Creative Commons Licence conditions.

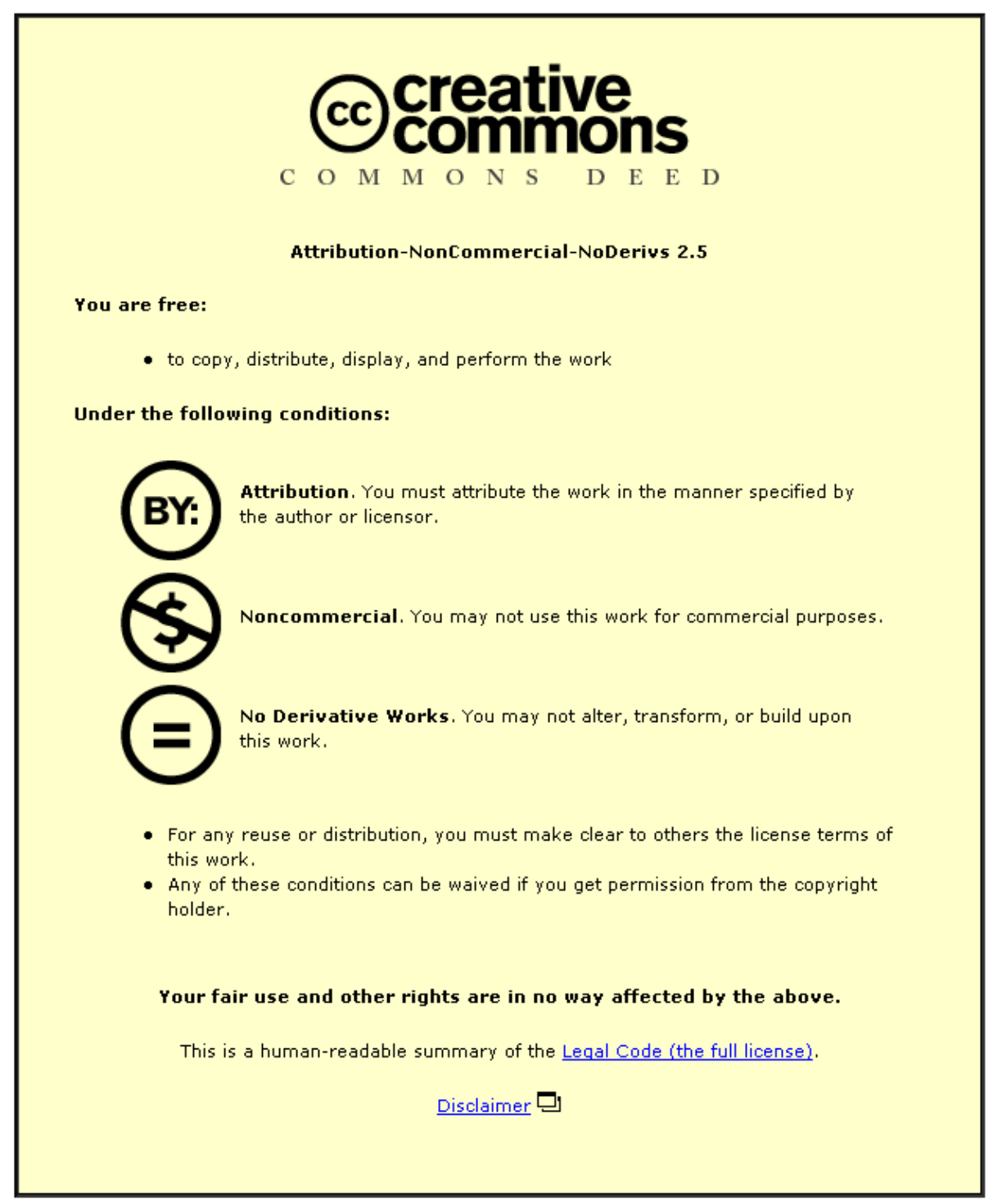

For the full text of this licence, please go to: http://creativecommons.org/licenses/by-nc-nd/2.5/ 


\title{
Aggregation in Colloidal Suspensions and its Influence on the Suspension Viscosity ${ }^{1}$
}

\author{
N. M. Kovalchuk ${ }^{a, b}$, I. Kuchin ${ }^{c}$, V. Starov ${ }^{a}$ and N. Uriev ${ }^{c}$ \\ ${ }^{a}$ Department of Chemical Engineering, Loughborough University, Loughborough, LE $113 T U, U K$ \\ ${ }^{b}$ Institute of Biocolloid Chemistry, National Academy of Sciences of Ukraine, pr. Vernadskogo 42, Kiev, 03142 Ukraine \\ ${ }^{c}$ Frumkin Institute of Physical Chemistry and Electrochemistry Russian Academy of Sciences, \\ Leninskii pr. 31, Moscow, 119991 Russia \\ e-mail:V.M.Starov@lboro.ac.uk \\ Received July 6, 2009
}

\begin{abstract}
Influence of aggregate formation on rheological properties of concentrated suspensions is discussed and reviewed. Methods of Stokesian and Brownian dynamic simulations of aggregation are discussed and the results of simulations for both Brownian and non-Brownian particles are presented.
\end{abstract}

DOI: $10.1134 / \mathrm{S} 1061933 X 10030117$

\section{INTRODUCTION}

Colloids are very important part of our everyday life. Technological processes in pharmaceutical, ceramic, food, mine industries, painting and coating as well as oil recovery often include processing and/or transportation of concentrated colloidal suspensions and emulsions and so their rheological properties become crucial for the performance optimisation.

Even for suspension composed of non-interacting hard spheres (HS) i.e. that, where colloidal interactions between particles are suppressed, rheological properties depend considerably on solid volume fraction and particle size distribution $[1,2]$.

At low solid volume fraction (dilute limit) every particle in suspension moves independently and suspension viscosity can be found from the Einstein relation as

$$
\eta=\eta_{0}\left(1+\frac{5}{2} \gamma\right)
$$

where $\eta$ is the effective suspension viscosity, $\eta_{0}$ is the viscosity of the dispersion medium, and $\gamma$ is the solid volume fraction.

As solid volume fraction grows, particles become involved in hydrodynamic interactions with each other resulting in the increase of viscosity, which can be described by virial expansion:

$$
\eta=\eta_{0}\left(1+k_{1} \gamma+k_{2} \gamma^{2}+\ldots\right)
$$

where $k_{1}=5 / 2$ as in Eq. (1). Second virial coefficient, $k_{2}$, depends on the forces acting between particles and on the particle shape. For spherical particles with hydrodynamic interactions only $k_{2}=6.2$ [3]. Unfortu-

\footnotetext{
${ }^{1}$ The article is published in the original.
}

nately, the range of applicability of Eq. (2) is rather limited as taking into account the second order term allows to estimate the viscosity of suspension with solid volume fraction up to $0.15-0.2$ with error $10 \%$ or higher [4].

After certain threshold solid volume fraction (ca. 0.25 for HS suspension [5]) suspension becomes nonNewtonian liquid with viscosity dependent on shear stress/rate imposed. Suspensions composed by hard spheres demonstrate mainly a shear thinning behaviour and dependence of viscosity on the shear stress is represented by Newtonian plateau at low shear followed by shear thinning and further by second plateau at high shear stresses $[2,5]$. The values of low and high shear viscosity can be calculated according to Dougherty-Krieger equation $[5,6]$ :

$$
\eta=\eta_{0}\left(1-\frac{\gamma}{\gamma_{\max }}\right)^{-[\eta] \gamma_{\max }},
$$

where $\gamma_{\max }$ is the maximum solid volume fraction corresponding to the viscosity divergence and $[\eta]$ is the intrinsic viscosity. Both $\gamma_{\max }$ and [ $\left.\eta\right]$ should be considered as fitting parameters with value of $[\eta]$ being close to 2.5 and $\gamma_{\max }$ for low shear viscosity being smaller than that for high shear viscosity [6]. It is usually accepted that for the case of high shear viscosity $\gamma_{\max }$ is equal to the close packing limit $[2,7]$.

At high enough volume fractions (above 0.45 ) the shear thinning is followed sometimes by shear thickening at further increase of the shear rate [1,8].

Colloidal interactions between particles (either attraction or repulsion) result in an increase of viscosity. Repulsive forces, either steric or electrostatic, sometimes are modelled through the increase of the effective radius of the particles and then using Eq. (2) 
or (3). For Eq. (2) in this case $k_{2}=2.5+\frac{3}{40}\left(2 a_{\text {eff }} / a\right)^{5}$ and for Eq. (3) $\gamma_{\text {eff }}=\gamma\left(a_{\text {eff }} / a\right)^{3}[2,9]$, where $a$ is the particle radius.

Note, Eq. (1) is supposed to be valid for any particles size distribution. If, however, interactions between particles in suspension cannot be neglected, than particles size distribution should be taken into account. Polydisperse suspension has lower viscosity than monodisperse one at identical solid volume fraction [1013] even if the mean particles size is kept constant [14].

The most complicated situation occurs if attractive forces prevail in the system and particles aggregate in the primary or, especially, in the secondary potential well, because in the case of aggregated suspension the aggregates size distribution and the microstructure of suspension depend on the shear rate imposed $[2,7]$. The aim of this review is to present the recent theoretical and experimental achievements in the influence of cluster formation on the rheological properties of suspensions, namely on the suspension viscosity as well as the methods of direct numerical simulations of the perikinetic and ortokinetic aggregation taking into account colloidal and hydrodynamic forces acting in the system.

The paper is organised as follows. Section 2 discusses the effect of cluster formation on the suspension viscosity. Sections 3 and 4 describe correspondingly the methods enabling modelling the cluster formation in suspensions composed of submicron particles undergoing the Brownian motion and coarser particles, where Brownian motion can be neglected. Finally, conclusions are presented in section 5.

\section{CLUSTERING AND VISCOSITY OF CONCENTRATED SUSPENSIONS}

If the attractive forces between particles prevail in a suspension, they cause the aggregation processes. The final aggregated state depends on the strength and range of interparticle forces, the properties of dispersion medium, temperature, the solid volume fraction and external forces acting on the particles in a suspension (gravity, electrical or magnetic fields, hydrodynamic flows). If interactions between particles are strong enough, the solid volume fraction is large and external forces provoking the breakage of bonds between particles (for example shear stress) are absent or small enough, then gel-like structures possessing visco-elastic properties are formed [2, 7]. Otherwise the suspension is composed of clusters of particles and demonstrates predominantly liquid-like behaviour. Therefore its rheological behaviour can be characterised quite adequately through the steady state viscosity. This case will be considered below.
Clusters usually have a fractal structure with power law dependency of mass on the radius of aggregate

$$
m=R_{\mathrm{g}}^{d_{\mathrm{f}}},
$$

where $m$ is the mass of cluster, $R_{\mathrm{g}}$ is the gyration radius, $1<d_{\mathrm{f}}<3$ is the mass fractal dimension [15-17]. The smaller is $d_{\mathrm{f}}$ the looser is the cluster. Obviously, the volume of cluster is larger than the total volume of particles included in cluster. That means, the clustering results in an increase of the effective solid volume fraction of suspension owing to dispersion medium entrapped inside the cluster and therefore one can expect an increase in the viscosity with the increase of the size of clusters as well as with the decrease of mass fractal dimension.

The clusters size can be controlled, for example, by variation of concentration of coagulant (dispersant). It is confirmed experimentally that while keeping constant solid volume fraction and shear rate imposed, the viscosity of suspension increases with the increase of the clusters size [18-20]. Direct microscopic observations of clusters performed in [21] during the viscosity measurements also confirmed this tendency.

As mentioned above, for flocculated suspensions the size, shape and structure of clusters and, therefore, the effective solid volume fraction as well as effective friction coefficient depend on the shear rate imposed. The increase in the shear rate results in formation of smaller and more compact aggregates due to both breakage and rearrangement processes [23-25], i.e. effective solid volume fraction decreases with the increase of the shear rate. The low shear rate plateau can be absent in weakly flocculated suspensions, as in this case the breakage of aggregates resulting in the decrease of the effective solid volume fraction begins already in the beginning of measurement [26].

Note the system equilibration after change of the shear rate imposed requires certain time. Thus the thixotropic behaviour should be expected for flocculated suspensions [9]. Indeed, thyxotropy has been observed in experimental studies on rheology of flocculated suspensions $[18,20]$.

Often the behaviour of suspension composed of clusters is qualitatively similar to the behaviour of suspension composed of hard spheres: viscosity demonstrates pseudo-Newtonian regions at low and high shear rates and shear thinning in between [9]. That is why the Dougherty-Krieger equation (3) can be applied to fit experimental data on the viscosity of flocculated suspensions with $\gamma$ being now the effective solid volume fraction [7, 22]. Otherwise the concept of the effective maximum solid volume fraction can be used [18, 19].

Below the applicability of Dougherty-Krieger Eq. (3) for calculation of viscosity of aggregated suspensions will be discussed in details following [27-31] where consideration is based on the Bruggemann's 
differential method for determination of effective properties of non-homogenous media [32].

Let us consider a suspension containing monodisperse spherical particles aggregated in clusters of various size with $n_{i}$ being a number of clusters containing $i$ particles and $\gamma_{i, \max }$ being an averaged packing density of single particles inside clusters $\left(\gamma_{1, \max }=1\right)$. Then the volume fraction of clusters containing $i$ particles is

$$
\gamma_{i}=\frac{V_{i}}{V}, \quad V_{i}=\frac{i v n_{i}}{\gamma_{i, \max }}, \quad i=1,2,3, \ldots,
$$

where $V$ is the volume of suspension, $V_{i}$ is the volume of cluster, $v=\frac{4 \pi}{3} a^{3}$ is the volume of the particle, $a$ is the radius of the particle. dition

Note, $\gamma_{i}$ should satisfy the mass conservation con-

$$
\sum_{i=1}^{\infty} \gamma_{i} \gamma_{i, \max }=\gamma
$$

where $\gamma=\frac{v N}{V}$ is the solid volume fraction in suspension composed by singlets only and $N$ is the number of particles in the volume $V$.

It is assumed in [27-31] that the viscosity of the suspension is completely determined by the cluster size distribution and the viscosity of dispersion medium $\eta_{0}$ :

$$
\eta\left(\eta_{0}, \gamma\right)=\Psi\left[\eta_{0}, \gamma_{1}, \gamma_{2}, \gamma_{3}, \ldots\right]
$$

At low volume fraction, $\gamma$, function $\Psi$ should obey Eq. (1), i.e.

$$
\Psi\left[\eta_{0}, \gamma_{1}, \gamma_{2}, \gamma_{3}, \ldots\right]=\Psi\left[\eta_{0}, 0,0,0, \ldots\right]+2.5 \sum_{i=1}^{\infty} \eta_{0} A_{i} \gamma_{i},
$$

where $\Psi\left(\eta_{0}, 0,0,0, \ldots\right)=\eta_{0}$ is the viscosity of dispersion medium, $A_{i}$ are deviations of friction coefficient of clusters from the corresponding value for single particle of the same diameter $\left(A_{1}=1\right)$, further referred to as friction coefficients for the simplicity. The friction coefficients can be calculated using the theory proposed in [33].

To derive the expression for the suspension viscosity in [27-31] some small number of clusters $\Delta n_{i} \ll n_{i}$ was marked randomly and the whole suspension was considered as a mixture of the marked clusters and dispersion medium containing the non-marked clusters. The viscosity of suspension composed of marked clusters was then compared to that given by Eq. (7) and following equation for the suspension viscosity was derived:

$$
\frac{d \eta}{d \gamma}=2.5 \eta\left[\frac{d\left(\bar{A} \frac{\gamma}{\bar{\gamma}_{\max }}\right)}{d \gamma}+\frac{\bar{A} \frac{\gamma}{\bar{\gamma}_{\max }}}{1-\frac{\gamma}{\bar{\gamma}_{\max }}} \frac{d\left(\frac{\gamma}{\bar{\gamma}_{\max }}\right)}{d \gamma}\right],
$$

where $\bar{A}=\frac{\sum_{i=1}^{\infty} A_{i} \gamma_{i}}{\sum_{i=1}^{\infty} \gamma_{i}}$ is the averaged resistance coefficient and $\bar{\gamma}_{\max }=\frac{\sum_{i=1}^{\infty} \gamma_{i, \max } \gamma_{i}}{\sum_{i=1}^{\infty} \gamma_{i}}$ is the averaged packing density.

If $\bar{A}$ is assumed to be independent of volume concentration then Eq. (9) is reduced to

$$
\frac{d \eta}{d \gamma}=\frac{2.5 \eta \bar{A}}{1-\frac{\gamma}{\bar{\gamma}_{\max }}} \frac{d\left(\frac{\gamma}{\bar{\gamma}_{\max }}\right)}{d \gamma}
$$

The solution of Eq. (10) is

$$
\frac{\eta(\gamma)}{\eta_{0}}=\frac{1}{\left(1-\frac{\gamma}{\bar{\gamma}_{\max }}\right)^{2.5 \bar{A}}} .
$$

Obviously (11) coincides with Dougherty-Krieger equation (3) with $[\eta]=\frac{2.5 \bar{A}}{\bar{\gamma}_{\max }}$, but the physical meaning of $\bar{\gamma}_{\max }$ in Eq. (11) is the averaged packing density of particles in the clusters. Eq. (11) enables to provide the good fit of the available experimental data on the viscosity of concentrated suspension with the reasonable parameters values (Fig. 1, [29]).

The above consideration shows clearly that clusters formation has an essential effect on the properties of suspension, particularly on its rheological behaviour.

In quiescent suspension initially composed of submicron particles the aggregation can occur due to Brownian motion. The most common and well understood case of the cluster formation is the aggregation of particles in the primary potential well. The aggregation kinetics is then either diffusion limited [34] or reaction limited [35] depending on the absence or presence of potential barrier before the primary potential well. More complicated case is the formation of stable clusters in equilibrium with singlets. The review of experimental and theoretical results on this topic is given in [36]. In suspensions composed of coarse particles, where the Brownian motion is negligible, the aggregation can be the result of particles collisions due to differential sedimentation or imposed motion with the velocity gradient, for example shearing. 


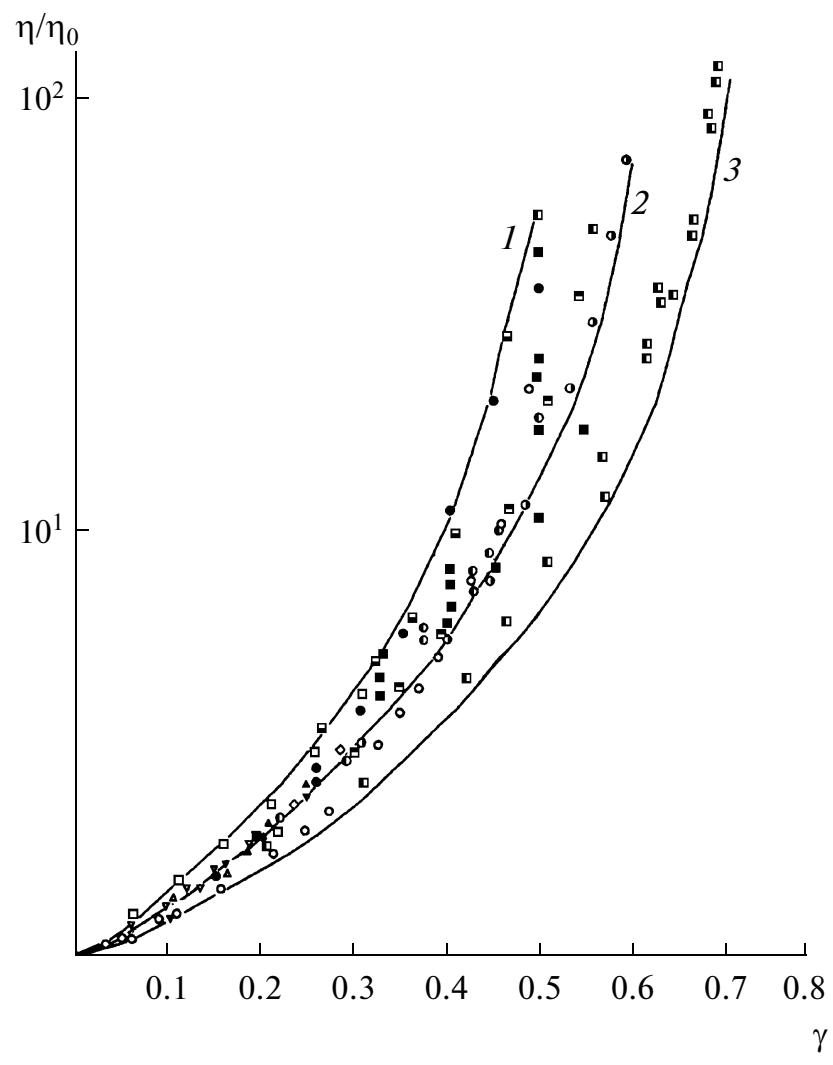

Fig. 1.Fitting of experimental data on relative viscosity as a function of volume concentration of dispersed particles with Eq. (11) [29]: $1-\bar{\gamma}_{\max }=0.56$ (close to simple cubic packing of particles inside clusters), $\bar{A}=0.72 ; 2-\bar{\gamma}_{\max }=$ 0.65 (close to cubic centered packing of particles inside clusters), $\bar{A}=0.67 ; 3-\bar{\gamma}_{\max }=0.73$ (close to hexagonal packing of particles inside clusters), $\bar{A}=0.61$.

The numerical methods are widely used to study the aggregation processes in suspensions [37]. Below we consider in more details two of the most broadly used direct numerical methods: the Brownian dynamic simulations applicable for particles in the submicron range and the Stokesian dynamic simulation for particles larger than $1 \mu \mathrm{m}$.

\section{CLUSTER FORMATION IN SUSPENSIONS OF BROWNIAN PARTICLES: DIRECT NUMERICAL SIMULATIONS}

In the Brownian dynamic method the dispersion medium is considered as continuous one with prescribed macroscopic properties, such as density and viscosity. The motion of particles is described by the system of Langevin equations [38]:

$$
m \frac{d U_{i}}{d t}=-\sum_{j=1}^{3 N} \varsigma_{i j} U_{j}+\sum_{j=1}^{3 N} \alpha_{i j} f_{j}+\sum_{j=1}^{3 N} F_{i j},
$$

where $N$ is the number of particles in the system, $m=\frac{4}{3} \pi a^{3}\left(\rho_{\mathrm{p}}+0.5 \rho_{\mathrm{l}}\right)$ is the mass of the particle (including the added mass), $a$ is the particle radius, $\rho_{\mathrm{p}}$ is the density of the particle material, $\rho_{1}$ is the density of the dispersion medium, $U$ is the particle velocity with $U_{1}$ being the $x$-component of velocity of first particle, $U_{2}$ being the $y$-component of velocity of first particle and so on, $\varsigma_{i j}$ stay for the element of the hydrodynamic resistance matrix, $F_{i j}$ are the components of colloidal forces and $\hat{\alpha}_{i j} f_{j}$ represents the $i$-th component of the Brownian forces, with $f_{j}$ being a random quantity, normally distributed, with

$$
\begin{gathered}
\left\langle f_{i}\right\rangle=0, \\
\left\langle f_{i}(t) f_{j}\left(t^{\prime}\right)\right\rangle=2 \delta_{i j} \delta\left(t-t^{\prime}\right) .
\end{gathered}
$$

The elements of matrices $\alpha_{i j}$ and $\varsigma_{i j}$ are correlated through the fluctuation-dissipation theorem [39]:

$$
\varsigma_{i j}=\frac{1}{k T} \sum_{l} \alpha_{i l} \alpha_{l j}
$$

where $k$ is the Boltzmann constant, $T$ is the absolute temperature.

Equation (12) can be solved on the diffusion time scale as it is proposed in [39]. In this case simulations should be performed with the time step larger than the momentum relaxation time $d t \gg t_{\mathrm{p}}=\frac{m}{\varsigma}$ and random walk of particles in suspension is described by the set of equations

$$
r_{i}=r_{i}^{0}+\sum_{j=1}^{3 N} \frac{\partial D_{i j}^{0}}{\partial r_{j}} \Delta t+\sum_{j=1}^{3 N} \frac{D_{i j}^{0} F_{i j}^{0}}{k T} \Delta t+R_{i}(\Delta t),
$$

where $D_{i j}$ is the tensor of particles diffusion coefficients, $R_{i}$ is the random displacement due to Brownian force acting on the particle and superscript 0 stay for the values taken from the preceding time step. The imposed shear could be included in this approach as well [40].

To simplify further the simulations based on Eq. (16) some authors neglect the hydrodynamic interactions between particles and use scalar $D_{0}$ - the diffusion coefficient of freely moving particle instead of tensor $D_{i j}$ [41]. The correctness of this approximation is, however, not obvious.

Another approach for numerical simulations based on Eq. (12) is proposed in [42, 43], where the simulations have been performed on the time scale $d t \ll t_{\mathrm{p}}=\frac{m}{\zeta}$ and, therefore, inertial term in the left hand side of Eq. (12) was taken into account. In the 
frameworks of this approach the particle velocity retains its physical meaning, i.e. the mean kinetic energy of particles becomes equal to the energy of thermal motion and so can be used as a control parameter assuring that there is no artificial pumping/sinking of energy in the system during the simulation. The latter is crucially important for the direct simulation of reversible aggregation in order to provide the correct life time of the particles inside clusters.

In [42, 43] Brownian dynamics computer simulations of reversible aggregation in 2D colloidal suspensions were performed on the base of Eq. (12) allowing for Brownian, hydrodynamic and colloidal forces and assuming pairwise additivity for all those forces, including hydrodynamic. The interactions with only nearest neighbours were taken into account. Interactions of the particle with each of its nearest neighbours were considered separately in the local co-ordinate system connected with the pair, whereupon all forces acting on the particle were summarised.

To model the colloidal interaction between particles, the simple dependency of colloidal force per unit area between two parallel flat surfaces, $\Phi(h)$, shown in Fig. 2a was adopted in [42, 43]. The latter however, represents the main features of the real force: the presence of both repulsion and attraction. The colloidal force between particles (Fig. 2b) was then calculated according to the Derjaguin's approximation $[44,45]$.

The matrix of hydrodynamic interactions between two particles in the local coordinate system with $x$-axis coinciding with the line connecting the particles centres used in $[42,43]$ was deduced as follows:

$$
\hat{\varsigma}=\left(\begin{array}{cccc}
\varsigma+\varsigma_{x} & 0 & -\varsigma_{x} & 0 \\
0 & \varsigma+\varsigma_{y} & 0 & -\varsigma_{y} \\
-\varsigma_{x} & 0 & \varsigma+\varsigma_{x} & 0 \\
0 & -\varsigma_{y} & 0 & \varsigma+\varsigma_{y}
\end{array}\right)
$$

where

$$
\varsigma=6 \pi \eta_{0} a
$$

is the hydrodynamic resistance to the motion of a free particle whereas $\varsigma_{x}$ and $\varsigma_{y}$, stay for hydrodynamic interactions between particles. At the small separation between the particles surfaces $h \leq 0.1 a \varsigma_{x}$ and $\varsigma_{y}$ were calculated according to the lubrication approximation according to [46]. The hydrodynamic interactions at separations $h>2.5 a$ were neglected and the interaction forces for $0.1 a \leq h \leq 2.5 a$ were fitted providing a smooth transition between forces at $h \leq 0.1 a$ and 0 at $h=2.5 a$.
Force per unit area, dyn $/ \mathrm{cm}^{2}$

(a)

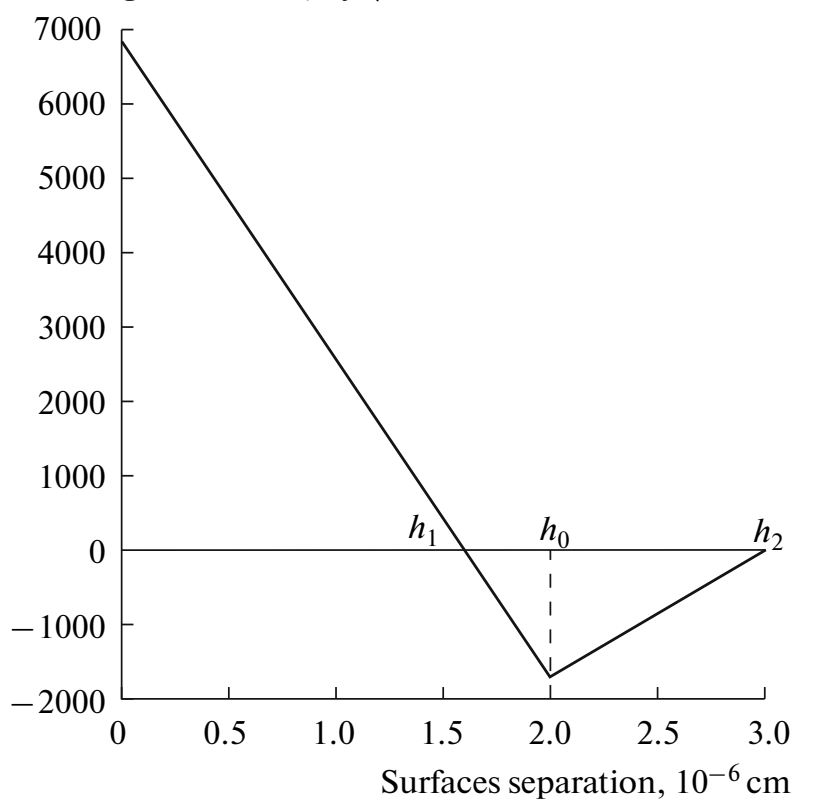

Interparticle force, $10^{-7} \mathrm{dyn}$

(b)

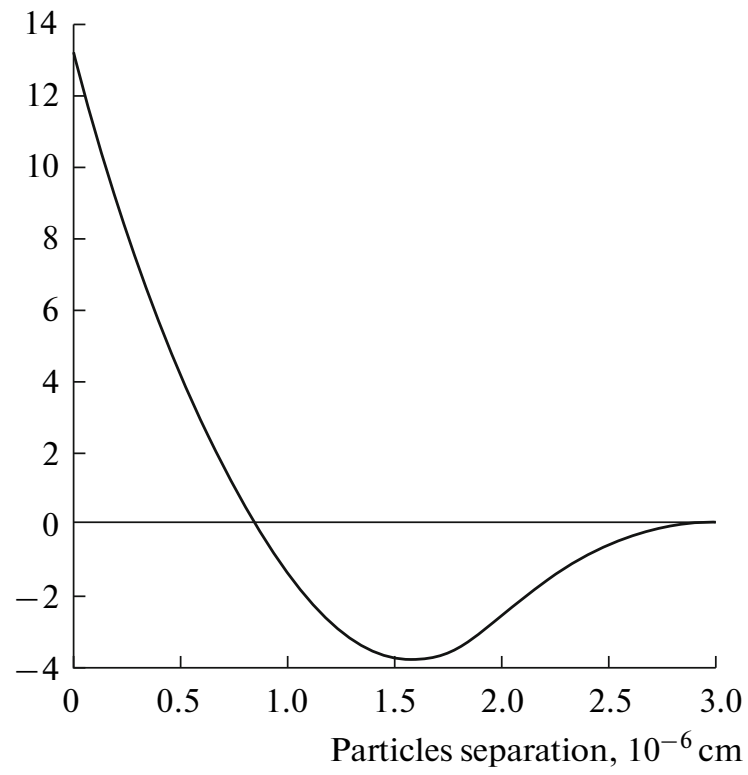

Fig. 2. Colloidal interaction between particles used in the computer simulations: (a) - the normal force per unit area between two parallel flat surfaces, (b) - force of interaction between particles: $h_{1}=1.6 \times 10^{-6} \mathrm{~cm}, h_{0}=2.0 \times 10^{-6} \mathrm{~cm}$, $h_{2}=3.0 \times 10^{-6} \mathrm{~cm}, U_{\min }=10 \mathrm{kT}$.

It has been shown in $[42,43]$ that the matrix of Brownian coefficients has the same form as the matrix of hydrodynamic coefficients:

$$
\hat{\alpha}=\left(\begin{array}{cccc}
\alpha_{11} & 0 & \alpha_{13} & 0 \\
0 & \alpha_{22} & 0 & \alpha_{24} \\
\alpha_{13} & 0 & \alpha_{11} & 0 \\
0 & \alpha_{24} & 0 & \alpha_{22}
\end{array}\right),
$$




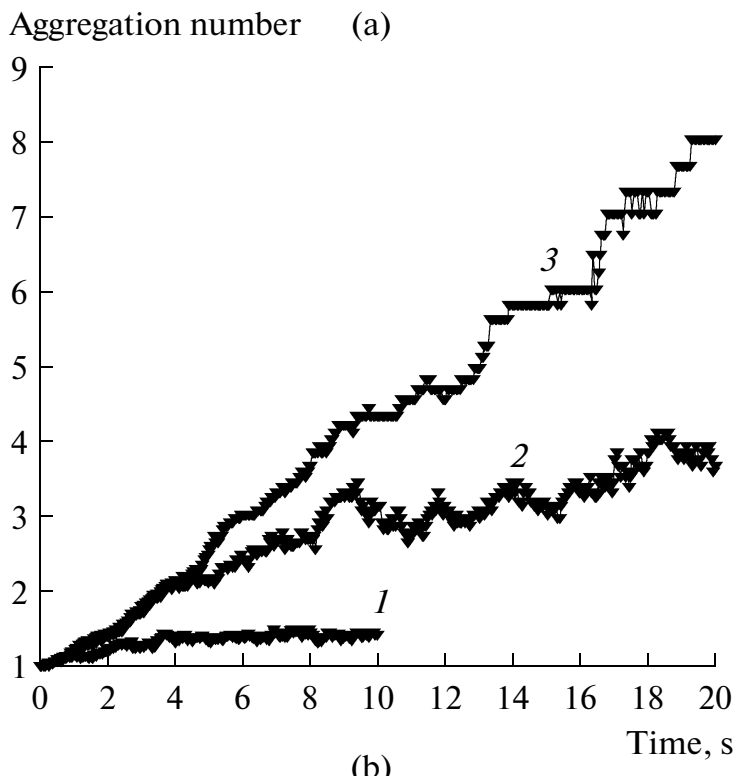

Number fraction of singlets

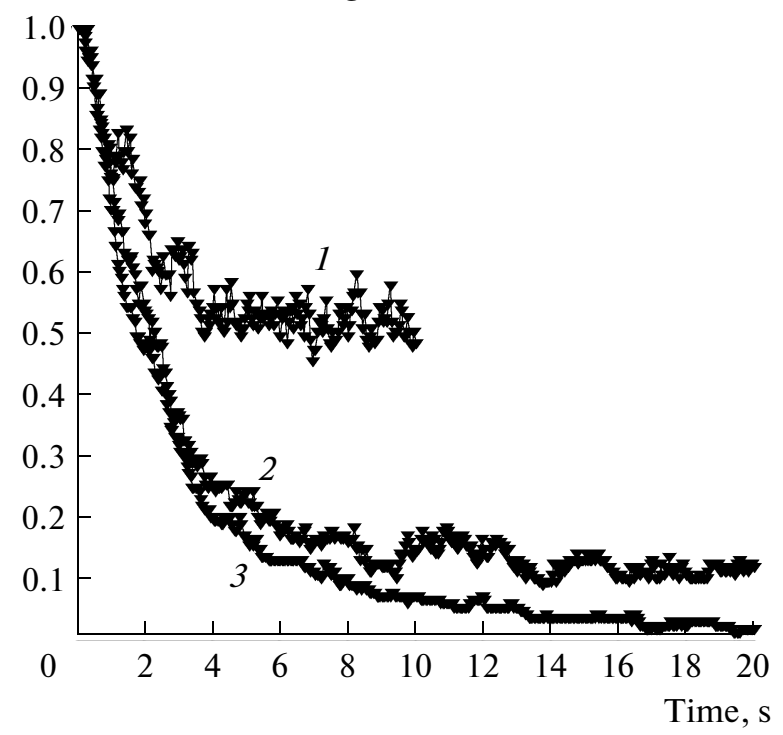

Fig. 3. Aggregation kinetics in colloidal suspension with 2D solid fraction 0.32 and parameters of potential well: $h_{1}=1.6 \times 10^{-6} \mathrm{~cm}, h_{0}=2.0 \times 10^{-6} \mathrm{~cm}, h_{2}=1.0 \times 10^{-5} \mathrm{~cm}$, $1-U_{\min }=3 k T, 2-U_{\min }=6 k T, 3-U_{\min }=20 k T$; (a) aggregation number vs time, (b) - number fraction of singlets vs time.
The components of random forces $f_{i}$ obeying Eqs. (18), (19) were modelled in numerical simulations as [47, 48]:

$$
f_{i}=\sqrt{\frac{2}{d t}} R_{\mathrm{ND}},
$$

where $d t$ is the time step used in computer simulations, $R_{\mathrm{ND}}$ is a random number from a normal distribution with the mean value equal to zero and the variance equal to 1 .

For validation of the mathematical model and numerical scheme, the value of diffusion coefficient of single particle and mean residence time of particle in potential well were found from the results of numerical simulations in $[42,43]$. The simulation results have shown good agreement with corresponding theoretical values.

Direct numerical simulations of clustering in colloidal suspensions performed in [42, 43] have shown the considerable importance of colloidal and hydrodynamic interactions on the aggregation kinetics and steady state cluster shape and size. The most important result is that at moderate depths of potential well the clusters of limited size in the dynamic equilibrium with singlets appears in the system, with mean cluster size depending on the depth of potential well and the range of colloidal forces. An example of the simulation results on aggregation kinetics is presented in Fig. 3. It shows that if potential well is deep enough $(20 k T)$ then the irreversible coagulation occurs in the system and aggregation number (mean number of particles in the cluster) steadily increases with time whereas the number of singlets decreases down to zero.

At weak colloidal interaction (the potential well depth $3 k T$ ) dynamic equilibrium establishes very quickly in the system. About a half of particles in the system remains free singlets and only small temporary clusters (mainly doublets and triplets) are formed. The mean aggregation number remains below 1.5.

When potential well depth increases to $6 k T$ clusters become much more stable, the mean number of particles in the cluster increases to about 4 . Still about $10 \%$ of particles remain as free singlets in this case. The particle rearrangement in the clusters was observed all the time over the duration of simulations. The snapshot pictures of clusters obtained in the course of numerical simulations for different potential well depths are presented in Fig. 4.

\section{STOKESIAN DYNAMIC SIMULATIONS OF SUSPENSION UNDER STEADY CONDITIONS AND UNDER SHEAR FLOW}

If a suspension is composed of particles larger than $1 \mu \mathrm{m}$ then the second term in the right hand side of Eq. (12) representing Brownian forces can be neglected and the simulations can be performed using the Stokesian dynamic method [49]. The results of Stokesian dynamic simulations of 2D concentrated suspension under shear flow are presented in [39]. 
(a)

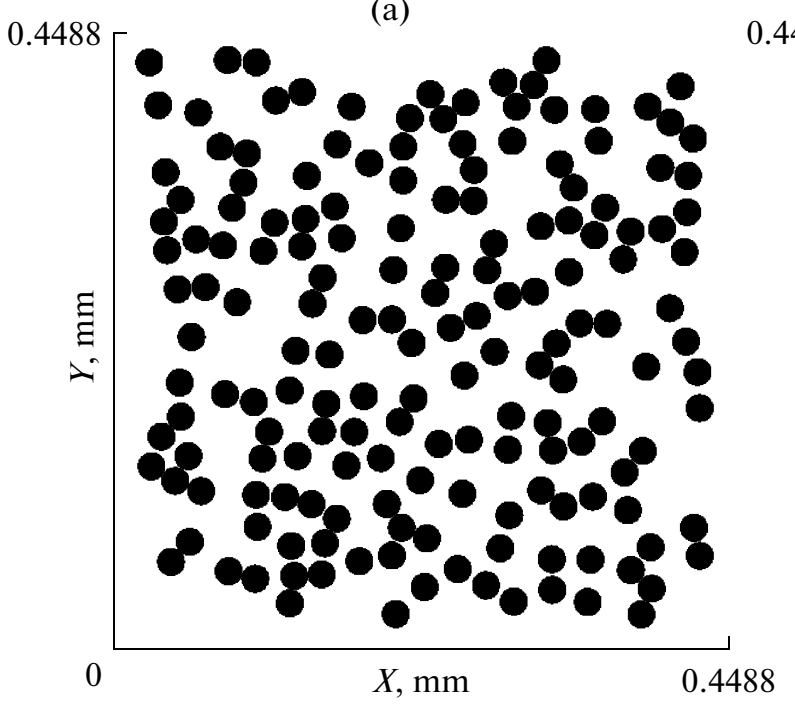

(b)

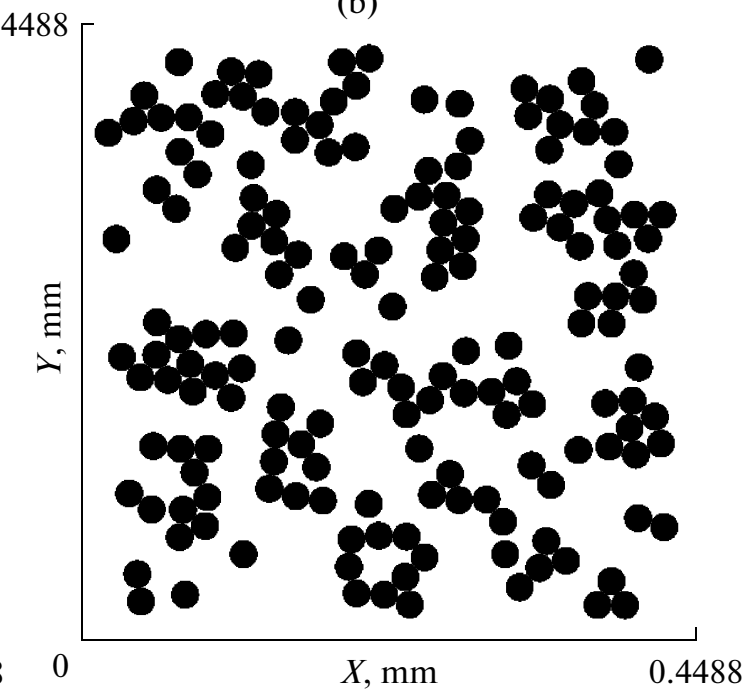

(c)

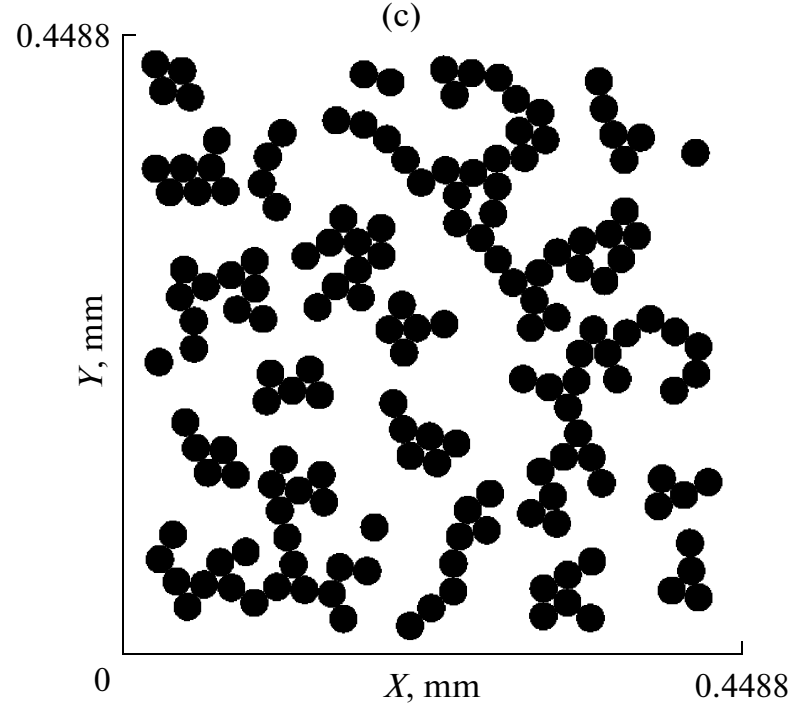

Fig. 4. Clusters in colloidal suspension with $2 \mathrm{D}$ solid fraction $\gamma=0.32$ and parameters of potential well: $h_{1}=1.6 \times 10^{-6} \mathrm{~cm}$, $h_{0}=2.0 \times 10^{-6} \mathrm{~cm}, h_{2}=1.0 \times 10^{-5} \mathrm{~cm}$; (a) $-U_{\min }=3 \mathrm{kT}$, (b) $-U_{\min }=6 \mathrm{kT}$, (c) $-U_{\min }=20 \mathrm{kT}$.

The colloidal interaction were modelled in [39] using function $F(h)$ similar to the real interaction curve having three intersection points with abscissa axis corresponding to equilibrium positions as [50]:

$$
F(h)=C \frac{\left(h-h_{1}\right)\left(h-h_{2}\right)\left(h-h_{3}\right)}{h^{5}},
$$

where $h_{1}, h_{2}, h_{3}$ - equilibrium positions in which the force is equal to zero; $C$ - a constant depending on the particles sizes and physical-chemical properties of components of dispersion.

The drag force on the free moving particle was calculated in [39] according to Stokes law accounting for screening effect:

$$
F^{\mathrm{st}}=-6 \pi \eta_{0} a\left(U-k U_{0}\right),
$$

where $U_{0}$ is the undisturbed velocity of dispersion medium in the particle centre and $k$ is the screening coefficient allowing for a screening of one particle by another in the flow of liquid. In general $k$ depends on the gap $h$ between particles surfaces and on the ratio of radii of screening $\left(a_{1}\right)$ and screened $(a)$ particles. In [39] it was accepted that $k=0.3$ in the case of screening and $k=1$ otherwise.

Hydrodynamic interactions between particles were calculated in [39]:

$$
\begin{gathered}
F_{i, j}^{\mathrm{hydr}}=-6 \pi \eta_{0} a\left(U_{i}-U_{j}\right) \quad \text { at } h \gg a, \\
F_{i, j}^{\mathrm{hydr}}=-\frac{\pi\left(U_{i}-U_{j}\right) a^{2}}{12 \beta^{2} \eta_{0}} \\
\times\left[\left(h+6 \beta \eta_{0}\right) \ln \left(1+\frac{6 \beta \eta_{0}}{h}\right)-\left(a+6 \beta \eta_{0}\right) \ln \left(1+\frac{6 \beta \eta_{0}}{a}\right)\right](25) \\
\text { at } h \ll a,
\end{gathered}
$$


(a) $t=0 \mathrm{~s}$

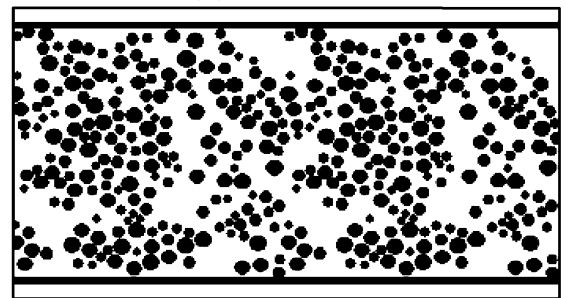

(c) $t=0.1 \mathrm{~s}$

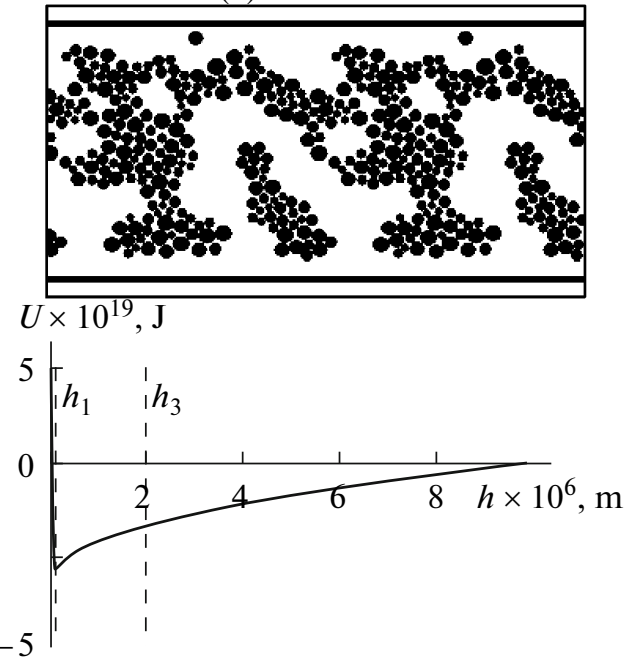

(b) $t=0.03 \mathrm{~s}$

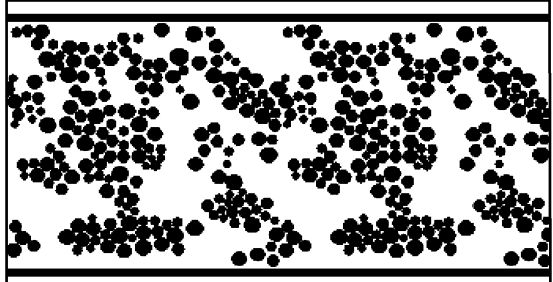

(d) $t=0.4 \mathrm{~s}$

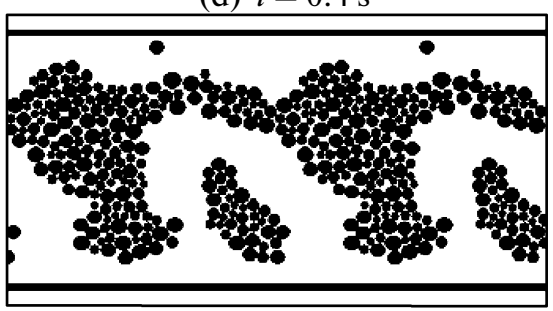

Fig. 5. Coagulation of suspension without imposed shear $(N=200 ; \gamma=35 \% ; a=0.5-1 \mu \mathrm{m})[22]$.

(a)

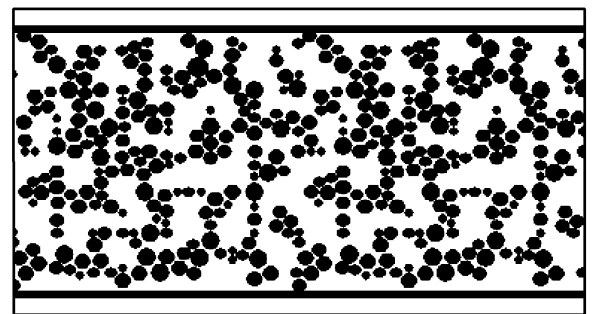

(c)

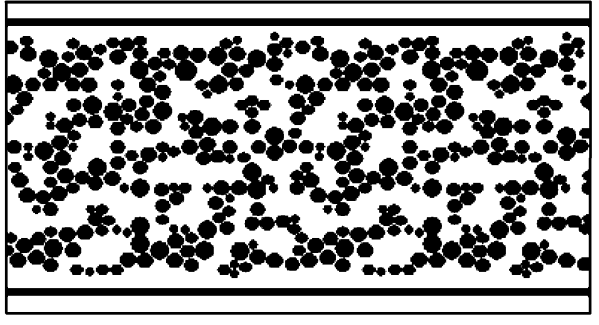

(b)

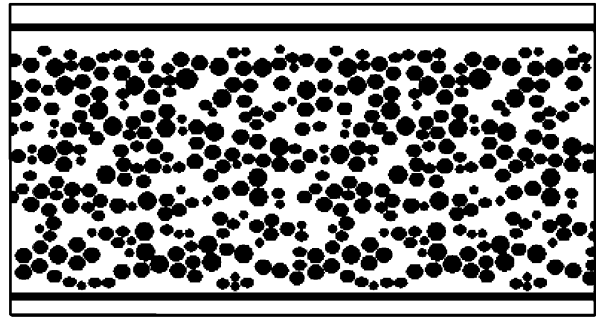

$U \times 10^{13}, \mathbf{J}$

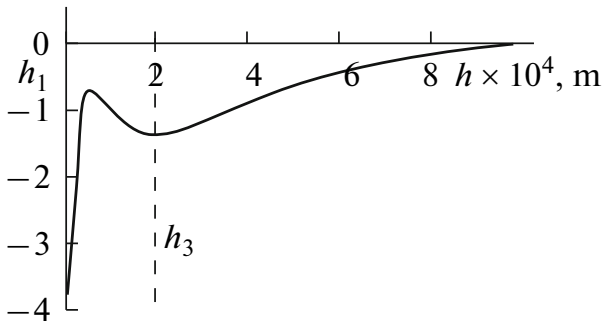

Fig. 6. Thixotropic behavior of suspension $(N=200 ; \gamma=40 \% ; a=0.5-1 \mu \mathrm{m})$ : (a) initial structure under static conditions; (b) structure destruction under the vibration; (c) initial structure recovery after stopping of vibration [22].

where $\beta$ is coefficient of liquid slippage on the particles surface. The gravity and Archimedean forces were neglected in [39].

Simulations performed in [39] for polydisperse suspensions with particles radius $2.5-50 \mu \mathrm{m}$ and $2 \mathrm{D}$ solid fraction 20-40\% under static conditions (without shear imposed) have shown that if a potential curve of particles interaction has a single well-expressed minimum, the system is unstable and coagulates with formation of the large and dense aggregates (Fig. 5). At a 
(a)

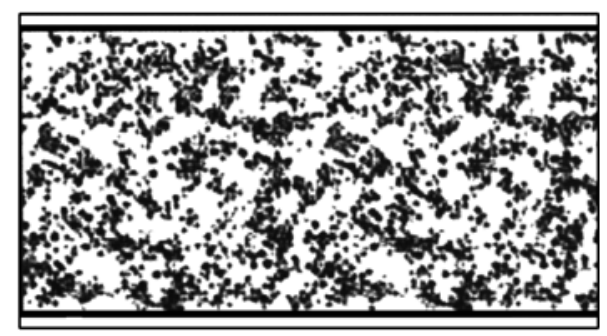

(c)

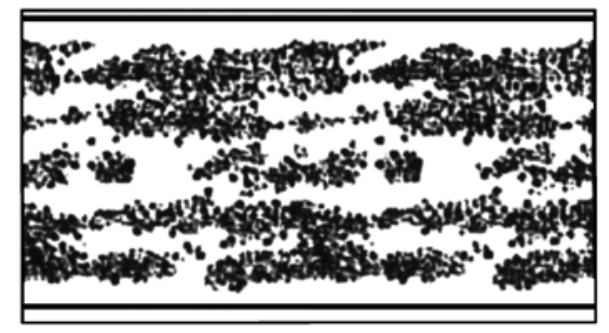

(b)

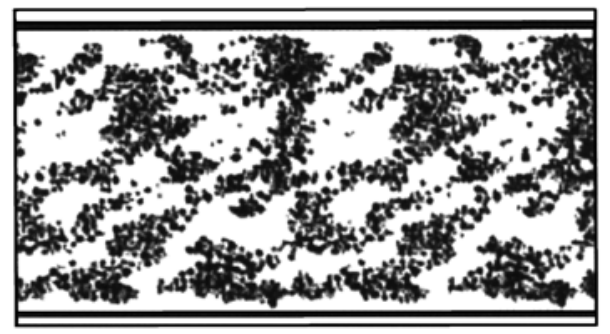

(d)

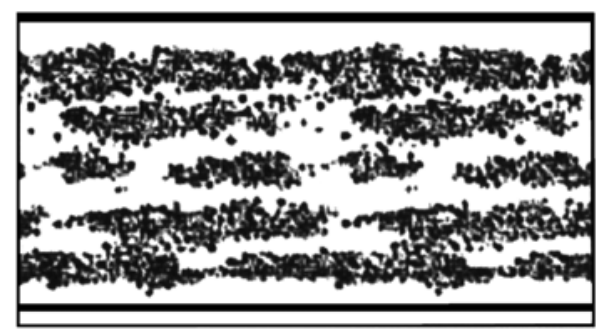

Fig. 7. Formation of the layered structure at flow of dispersion $\left(N=800\right.$; shear rate $\left.-85 \mathrm{~s}^{-1} ; \gamma=35 \% ; a=0.5-1 \mu \mathrm{m}\right)$. States of the systems at different points of time after start of flow, s: (a) 0 , (b) 0.05 , (c) 0.3 , and (d) 0.5 .

certain relation between attractive and repulsive forces and in certain interval of 2D solid fractions (as a rule at $40 \%$ ) the gel-like microstructure is formed in suspension (Fig. 6a). The larger the depth of both primary and secondary potential wells, the more expressed is the cell structure in the suspension and the higher a rate of its formation. The structure can be easily destroyed, for example, by imposed vibrations (Fig. $6 \mathrm{~b}$ ), but it recovers after vibrations is terminated (Fig. $6 \mathrm{c})$, i.e. dispersion demonstrates thixotropic behavior.

Under dynamic conditions of imposed shear flow the simulations performed in [39] displayed the formation of the layered structures (Fig. 7) observed earlier in experiments $[53,54]$. According to the simulation results [39] the formation of layers and their structure depend considerably on the strength and the range of colloidal forces. Similarly to the static case, the layered structures formed under dynamic conditions can be destroyed by supplying additional energy to the system in the form of external mechanic perturbations (e.g. vibration).

\section{CONCLUSIONS}

Colloidal interactions between particles composing a suspension have a considerable influence on the suspension properties. In particular, the rheological behaviour of suspension depends on its aggregation state. It has been shown that the viscosity of an aggregated suspension can be described by the equation similar to well known Dougherty-Krieger equation with a parameter having a clear physical meaning of the averaged packing density of particles in the clus- ters. Equation describes very well the experimental data available.

The direct numerical simulations using Brownian and Stokesian dynamic methods are a powerful tool for the study of aggregation processes enabling the comprehensive analysis of aggregation kinetics. In the case of Brownian particles the interplay between colloidal, Brownian and hydrodynamic forces results either in irreversible aggregation or establishment of dynamic equilibrium between aggregates and single particles. The formation of either large aggregates or gel-like structures was found by Stokesian dynamic simulation on suspensions composed by large particles $(>1 \mu \mathrm{m})$ with large solid fractions under static conditions. Formation of layered structures, similar to those observed in experiments, was predicted under dynamic conditions of imposed shear.

\section{ACKNOWLEDGMENT}

V.M. Starov and N.M. Kovalchuk would like to acknowledge the financial support from the Engineering and Physical Sciences Research Council, UK (Grant EP/C528557/1).

\section{REFERENCES}

1. Larson, R.G., The Structure and Rheology of Complex Fluids, New York-Oxford: Oxford Univ. Press, 1999.

2. Quemada, D. and Berli, C., Adv. Colloid Interface Sci., 2002, vol. 98, p. 51.

3. Batchelor, G.K., J. Fluid. Mech., 1977, vol. 83, p. 97.

4. Thomas, D.G., J. Colloid Sci., 1965, vol. 20, p. 267. 
5. Krieger, I.M., Adv. Colloid Interface Sci., 1972, vol. 3, p. 111 .

6. Krieger, I.M. and Dougherty, T.J., Trans. Soc. Rheol., 1959, vol. 3, p. 137.

7. Perez, M., Barbe, J.C., Neda, Z., et al., Acta Mater., 2000, vol. 48, p. 3773.

8. Hoffman, R.L., Trans. Soc. Rheol., 1972, vol. 16, p. 155 .

9. Tadros, T.F., Adv. Colloid Interface Sci., 1996, vol. 68, p. 97.

10. Jeffrey, D.J. and Acrivos, A., AIChE J., 1976, vol. 22, p. 417.

11. D'Haene, P. and Mewis, J., Rheol. Acta, 1994, vol. 33, p. 165.

12. Mewis, J., Int. J. Miner. Process., 1996, vols. 44-45, p. 17.

13. Nunez, G.A., Sanchez, G., Gutierrez, X., et al., Langmuir, 2000, vol. 16, p. 6497.

14. Lukham, P.F. and Ukeje, M.A., J. Colloid Interface Sci., 1999, vol. 220, p. 347.

15. Burns, J.L., De Yan, Y., Jameson, G.J., and Biggs, S., Langmuir, 1997, vol. 13, p. 6413.

16. Jackson, G.A., Maffione, R., Costello, D.K., et al., Deep-Sea Res. I, 1997, vol. 44, p. 1739.

17. Filippov, A.V., Zurita, M., and Rosner, D.E., J. Colloid Interface Sci., 2000, vol. 229, p. 261.

18. Harzallah, O.A. and Dupuis, D., Rheol. Acta, 2003, vol. 42, p. 10 .

19. Chen, S., Oye, G., and Sjoblom, J., J. Dispersion Sci. Technol., 2007, vol. 28, p. 845.

20. Shen, Z.-G., Chen, J.-F., Zou, H.-K., and Yun, J., Colloids Surf., A, 2004, vol. 244, p. 61.

21. Hamberg, L., Walkenstrom, P., Stading, M., and Hermansson, A.-M., Food Hydrocolloids, 2001, vol. 15, p. 139.

22. Quemada, D., Eur. Phys. J. AP, 1998, vol. 1, p. 119.

23. Selomulya, C., Bushell, G., Amal, R., and Waite, T.D., Langmuir, 2002, vol. 18, p. 1974.

24. Selomulya, C., Bushell, G., Amal, R., and Waite, T.D., Int. J. Miner. Process., 2004, vol. 73, p. 295.

25. Rulyov, N.N., Korolyov, V.Y., and Kovalchuk, N.M., Miner. Process. Extract. Metall., 2009, vol. 118, p. 175.

26. Doi, M., Kawaguchi, M., and Kato, T., Colloids Surf., $A, 2002$, vol. 211, p. 223.

27. Zhdanov, V.G. and Starov, V.M., Colloid J., 1998, vol. 60 , p. 771 .

28. Starov, V. and Zhdanov, V., Colloids Surf., A, 2001, vol. 192, p. 363.

29. Starov, V., Zhdanov, V., Meireles, M., and Molle, C., Adv. Colloid Interface Sci., 2002, vol. 96, p. 279.
30. Starov, V.M. and Zhdanov, V.G., J. Colloid Interface Sci., 2003, vol. 258, p. 404.

31. Starov, V.M. and Zhdanov, V.G., Adv. Colloid Interface Sci., 2008, vol. 137, p. 2.

32. Bruggemann, D.A., Ann. Phys., 1935, vol. 24, p. 636.

33. Perepelkin, P.V., Starov, V.M., and Filippov, A.N., Colloid J., 1992, vol. 54, p. 257.

34. Lin, M.Y., Lindsay, H.M., Weitz, D.A., et al., J. Phys.: Condens. Matter, 1990, vol. 2, p. 3093.

35. Lin, M.Y., Lindsay, H.M., Weitz, D.A., et al., Phys. Rev. A, 1990, vol. 41, p. 2005.

36. Kovalchuk, N., Starov, V., Langston, P., and Hilal, N., Adv. Colloid Interface Sci., 2009, vols. 147-148, p. 144.

37. Uriev, N.B. and Kuchin, I.V., Adv. Colloid Interface Sci., 2007, vols. 134-135, p. 249.

38. Coffey, W.T., Kalmykov, Yu.P., and Waldron, J.T., The Langevin Equation. With Applications in Physics, Chemistry and Electrical Engineering, World Sci. Ser. in Contemporary Chemical Physics, vol. 11.

39. Ermak, D.L. and McCammon, J.A., J. Chem. Phys., 1978, vol. 69, p. 1352.

40. Silbert, L.E., Melrose, J.R., and Ball, R.C., Phys. Rev. E: Stat. Phys., Plasmas, Fluids, Relat. Interdiscip. Top., 1997, vol. 56, p. 7067.

41. Cordelair, J. and Greil, P., J. Eur. Ceram. Soc., 2004, vol. 24, p. 2717.

42. Kovalchuk, N., Starov, V., Langston, P., and Hilal, N., J. Colloid Interface Sci., 2008, vol. 325, p. 377.

43. Kovalchuk, N., Starov, V., Langston, P., and Hilal, N., Colloid J., 2009, vol. 71, p. 503.

44. Derjaguin, B.V., Churaev, N.V., and Muller, V.M., Surface Forces, New York: Consultants Bureau, 1987.

45. Israelachvili, J.N., Intermolecular and Surface Forces, London: Academic, 1992.

46. Cox, R.G., Int. J. Multiphase Flow, 1974, vol. 1, p. 343.

47. Turq, P., Lanteime, F., and Friedman, H.L., J. Chem. Phys., 1977, vol. 66, p. 3039.

48. Groot, R.D. and Warren, P.B., J. Chem. Phys., 1997, vol. 107, p. 4423.

49. Brady, J.F. and Bossis, G., Annu. Rev. Fluid Mech., 1988, vol. 20, p. 111.

50. Uriev, N.B., Cheremisov, A.V., and Tkachev, A.Y., Colloid J., 1999, vol. 61, p. 383.

51. Potanin, A.A., Uriev, N.B., and Muller, V.M., Colloid J., 1987, vol. 49, p. 802.

52. Potanin, A.A., Uriev, N.B., and Muller, V.M., Colloid J., 1988, vol. 50, p. 429.

53. Uriev, N.B., Physicochemical Foundations of the Technology of Disperse Systems and Materials, Moscow: Khimiya, 1988.

54. Uriev, N.B. and Potanin, A.A., Fluidity of Suspensions and Powders, Moscow: Khimiya, 1992. 